\section{Avaliação ultrassonográfica da tireoide, determinação da iodúria e concentração de iodo em sal de cozinha utilizado por escolares de Ribeirão Preto, São Paulo, Brasil}

\author{
Ultrasonographic evaluation of the thyroid, determination \\ of ioduria and iodine concentration in kitchen salt used \\ by schoolchildren in Ribeirão Preto, São Paulo, Brazil
}

Maria Lúcia D'Arbo Alves', Gláucia C. Duarte²,

Anderson Marliere Navarro ${ }^{3}$, Eduardo K. Tomimori ${ }^{4}$

\section{RESUMO}

Objetivo e métodos: Estudos prévios apontaram Ribeirão Preto, São Paulo, como área de carência limítrofe de iodo e, posteriormente, de iodação excessiva. Reavaliando seu estado iódico em 2007-2008, foram analisados 300 escolares entre 8 e 10 anos de idade, procedentes de três escolas de diferentes níveis socioeconômicos e realizadas dosagens de iodo urinário e da concentração de iodo no sal doméstico e ultrassonografia tireoidiana. Resultados e conclusão: A iodúria foi superior na escola com maior nível socioeconômico, comparada às outras, e a diferença foi significante aos 8 e 10 anos para os meninos $(p<0,0001$ e $p=0,0106$, respectivamente) e aos 8 e 9 anos para as meninas ( $p=0,0024$ e $p=0,0154$, respectivamente). As concentrações medianas de iodo em amostras de sal doméstico variaram entre 26,6 e $27,8 \mathrm{mg}$ iodo/kg e não foram diferentes nas escolas estudadas. À ultrassonografia, 15,6\% das tireoides examinadas apresentaram-se hipoecoicas, sugerindo que elas possam apresentar mudanças em sua arquitetura sem ainda alterar seus volumes. Arq Bras Endocrinol Metab. 2010;54(9):813-8

\section{Descritores}

Iodo urinário; volume tireoidiano; sal iodado; deficiência de iodo; escolares

\begin{abstract}
Objective and methods: Previous studies have pointed out that Ribeirão Preto, São Paulo, is an area of borderline iodine deficiency, later becoming a region of excessive iodination. In a reevaluation of the iodine status of the city in 2007-2008, 300 schoolchildren aged 8 to 10 years were evaluated, from 3 schools of 3 different socioeconomic levels who were submitted to the determination of iodine concentration in urine samples and in kitchen salt and thyroid ultrasound. Results and conclusion: Among boys and girls ioduria was higher in the school of highest socioeconomic level compared to the others, with a significant difference in the ages 8 and 10 years for boys with ranges : $p<0,0001$ and $p=0,0106$, respectively; and in the ages 8 and 9 years for girls with ranges: $p=0,0024$ and $p=0,0154$, respectively. Median iodine concentrations in samples of kitchen salt ranged from 26.6 to $27.8 \mathrm{mg}$ iodine $/ \mathrm{kg}$ and did not differ between the schools studied. Ultrasound evaluation revealed that $15,6 \%$ of thyroids examined were hypoechoic, suggesting that these glands may present changes in the architecture not reflected yet in their volume. Arq Bras Endocrinol Metab. 2010;54(9):813-8
\end{abstract}

\section{Keywords}

Urinary iodine; thyroid volume; iodated salt; iodine deficiency; schoolchildren
${ }^{1}$ Curso de Medicina, Faculdade de Medicina de Ribeirão Preto Universidade de Ribeirão Preto (Unaerp), Ribeirão Preto, SP, Brasil 2 Unidade de Tireoide, Universidade de São Paulo (USP), São Paulo, SP, Brasil

${ }^{3}$ Curso de Nutrição, Faculdade de Medicina de Ribeirão Preto, Universidade de São Paulo (FMRP-USP), Ribeirão Preto, SP, Brasil ${ }^{4}$ Unidade de Tireoide, USP, São Paulo, SP, Brasil
Correspondência para: Maria Lúcia D'Arbo Alves Rua Cerqueira César, 845, ap. 62 14010-130 - Ribeirão Preto, SP, Brasil

mldarbo@bol.com.br

Recebido em 25/Maio/2010 Aceito em 3/Nov/2010 


\section{INTRODUÇÃO}

A tireoide é a maior glândula do corpo humano, está localizada na porção anterior do pescoço e é acessível à palpação e a exame ultrassonográfico. No entanto, o método palpatório é impreciso e subjetivo. A partir das décadas de 1980 e 1990, a ultrassonografia consagrou-se como método de escolha para o cálculo do volume tireoidiano tanto em estudos científicos quanto na prática clínica. Em indivíduos normais, o volume tireoidiano é influenciado pelo peso, altura, superfície corporal, idade (até a terceira década de vida) e o consumo de iodo na dieta (1-5).

O iodo é um micronutriente presente no corpo humano em quantidades muito pequenas $\left(0,02285 \times 10^{-3} \%\right.$ do peso corporal do adulto) (6). Na espécie humana, constitui-se em substrato essencial da composição dos hormônios tireoidianos, tiroxina (T4) e tri-iodotironina (T3). Esses hormônios têm um papel determinante para o metabolismo de todas as células do organismo, nos processos de crescimento e desenvolvimento da maior parte dos órgãos, em especial do cérebro (7). As recomendações da Organização Mundial da Saúde (OMS), Fundo das Nações Unidas para a Infância (Unicef) e International Council for the Control of Iodine Deficiency Disorders (ICCIDD) para a ingestão de iodo diária são: $50 \mathrm{mg}$ para recém-nascidos (até 12 meses), $90 \mathrm{mg}$ para crianças de 13 meses a 6 anos, $120 \mathrm{mg}$ para escolares (7-12 anos), $150 \mathrm{mg}$ para adultos (após 12 anos) e 250 mg para gestantes e lactantes. Nessas circunstâncias, a excreção de iodo esperada é de 10 a 20 ug iodo/dL (ou 100 a $200 \mathrm{ug}$ de iodo por litro de urina).

A deficiência de iodo é a principal causa evitável de dano cerebral em fetos e crianças, assim como de retardo do desenvolvimento psicomotor. E se persiste com uma deficiência iódica severa, tem o bócio e o cretinismo como suas manifestações extremas. Outras alterações como hipotireoidismo subclínico, baixo rendimento escolar, aumento da mortalidade perinatal e estagnação socioeconômica ocorrem em regiões de deficiência leve ou moderada, constituindo verdadeiro obstáculo ao desenvolvimento das populações (7-14).

A ingesta excessiva de iodo, cronicamente, também é prejudicial, bloqueando as características funcionais e/ou desencadeando alterações como aumento da incidência de bócio, de casos de tireoidite crônica e de hipertireoidismo subclínico na população (15).

No Brasil, desde 1956 (Decreto $n^{\circ}$ 39.814), a iodação do sal vigora para todo o território nacional, e a partir de maio de 2003 (RDC n ${ }^{\circ}$ 130) o Ministério da Saúde passa a considerar próprio para o consumo humano o sal que contiver teor igual ou superior a $20 \mathrm{mg}$ até o limite máximo de $60 \mathrm{mg}$ de iodo por quilograma de produto $(16,17)$. Estudos realizados mostram que o estado de São Paulo é considerado uma área iodossuficiente $(18,19)$.

Na cidade de Ribeirão Preto, foram publicados trabalhos com resultados conflitantes. Alves e cols. avaliaram, em 1988, a iodúria em recém-nascidos, concluindo que essa amostra populacional excretava iodo abaixo do recomendado pela OMS (20). Em 2003, a iodúria superior a $300 \mathrm{ug} / \mathrm{L}$ em $79,0 \%$ das amostras, $17,3 \%$ de bócio pela palpação e positividade de $20,2 \%$ nos títulos de anticorpos antitireoidianos em crianças de escolas municipais de diferentes níveis socioeconômicos foram considerados elevados para as faixas etárias examinadas (21).

Nesse contexto, o presente estudo reavalia o atual consumo iódico entre crianças de 8 e 10 anos de escolas pesquisadas anteriormente na cidade de Ribeirão Preto, por meio da dosagem da iodúria, da concentração de iodo no sal de consumo doméstico e do volume tireoidiano pela ultrassonografia, verificando as possíveis consequências para a glândula tireoide.

\section{SUJEITOS E MÉTODOS}

Foram analisados 300 escolares de área urbana (138 meninos e 162 meninas), entre 8 e 10 anos de idade, em três diferentes escolas públicas em zona urbana desse município, designadas pela Secretaria Municipal de Educação de Ribeirão Preto como as mais representativas de classes socioeconômicas distintas dessa cidade:

Escola $A$ - classe socioeconômica mais elevada (98 crianças: 50 meninos e 48 meninas).

Escola $B$ - classe socioeconômica intermediária (84 crianças: 44 meninos e 40 meninas).

Escola $C$ - classe socioeconômica mais baixa (118 crianças: 44 meninos e 74 meninas).

Todos os escolares examinados apresentaram o termo de consentimento para participação no estudo, devidamente preenchido pelo seu responsável. O protocolo deste trabalho foi aprovado pelo Comitê de Ética em Pesquisa da Universidade de Ribeirão Preto, registrado sob o número 063/06.

Todos os participantes da amostra eram pré-puberes e foram classificados em três grupos (8 anos -9 anos - 10 anos). Realizaram-se as medidas antropométricas (peso em quilogramas e altura em centímetros), ultrassonografia de tireoide, coleta de amostra de urina e de sal de uso doméstico. A superfície corporal foi calculada por meio da fórmula: 


$$
S C\left(m^{2}\right)=\sqrt{\{[\operatorname{peso}(\mathrm{kg}) \text { xaltura }(\mathrm{cm})] / 3600\}}
$$

O exame ultrassonográfico de tireoide foi realizado por um único examinador (GD), usando equipamento Logig Book GE, com transdutor linear multifrequencial de alta resolução de 7,5 a $10 \mathrm{MHz}$. O volume foi calculado a partir da fórmula: eixo longitudinal $\mathrm{x}$ eixo anteroposterior x 0,52 de cada um dos lobos e istmo (22). O volume total foi a soma dos dois lobos e o istmo. Avaliou-se a ecogenicidade da tireoide, quantificada por análise visual de escala de cinza, classificando-a em normal, leve, moderada ou acentuadamente hipoecoica, comparando-se o parênquima tireoidiano com a glândula submandibular e os músculos cervicais adjacentes. Adicionalmente, houve a descrição de eventuais achados ultrassonográficos.

Foram colhidas amostras casuais urinárias em potes plásticos identificados, para a avaliação da iodúria, quantificada pela reação de Sandell-Kolthoff (23), precedida pela digestão da urina pelo persulfato de amônio (24). Os valores foram expressos em microgramas por litro (ug/L) de urina e classificados, de acordo com o ICCI$\mathrm{DD}$, em suficiência de iodo se a mediana se apresentava $>100 \mathrm{ug} / \mathrm{L}$ e $<300 \mathrm{ug} / \mathrm{L}$, insuficiência iódica se a mediana estava $<100 \mathrm{ug} / \mathrm{L}$ e excessiva iodação para valores medianos de iodúria $>300 \mathrm{ug} / \mathrm{L}(25)$. Solicitou-se às crianças que trouxessem de casa amostra de 3 colheres de sopa de sal de uso doméstico (sal de cozinha) para dosagem do teor de iodo por meio de titulação, com valor de referência entre $20-60 \mathrm{mg}$ de iodo $/ \mathrm{kg}$ de sal.

\section{ANÁLISE ESTATÍSTICA}

Analisaram-se descritivamente os dados e avaliaram-se as diferenças entre cada uma das escolas (A, B e C), em cada uma das idades $(8,9$ e 10 anos $)$ para as variáveis do estudo (volume tireoidiano, iodúria e concentração de iodo em amostras de sal de uso doméstico). Os dados foram analisados mediante um nível de significância adotado de $5 \%(\alpha=0,05)$. Dessa forma, os valores calculados da probabilidade de erro $(\mathrm{p})$, quando $\leq 0,05$, foram considerados estatisticamente significantes e, quando $>0,05$, foram tomados como não significantes. Utilizou-se análise de variância (ANOVA) $(26,27)$, por meio do software SAS $^{\circledR}$ 9.0, utilizando a PROC GLM. Para as comparações, foram utilizados contrastes ortogonais baseados na distribuição t. Para o cálculo de correlação, utilizou-se o coeficiente de correlação de Pearson (28). A avaliação ultrassonográfica da tireoide, por meio da ecogenicidade considerada normal ou diminuída, foi submetida a teste $\mathrm{Z}$, determinando se a diferença entre a média da amostra (ecogenicidade diminuída) e a média da população (ecogenicidade normal) é grande o suficiente para ser estatisticamente significante (29).

\section{RESULTADOS}

\section{Medidas antropométricas: peso, altura e superfície corporal}

Os valores médios, desvios-padrão e significância estão representados na tabela 1 . Não houve diferença estatística na variável peso e superfície corporal entre as diferentes faixas etárias ou entre os sexos. A altura média dos meninos das três escolas foi estatisticamente diferente $(\mathrm{p}<0,05)$, mas não foi diferente entre as meninas.

Tabela 1. Média e desvio-padrão dos valores antropométricos dos escolares de Ribeirão Preto

\begin{tabular}{lccccccc}
\hline & \multicolumn{2}{c}{ Meninos } & & \multicolumn{2}{c}{ Meninas } & \multirow{2}{*}{ Teste t } \\
\cline { 2 - 3 } & Média & DP & & Média & DP & \\
\hline Idade & 8,91 & 0,84 & & 9,16 & 0,77 & 0,008 \\
Peso & 35,33 & 9,35 & & 34,53 & 8,98 & 0,454 \\
Altura & 1,37 & 0,08 & & 1,36 & 0,08 & 0,334 \\
S.C. & 1,15 & 0,17 & & 1,14 & 0,17 & 0,465 \\
VolT & 2,25 & 0,64 & & 2,14 & 0,66 & 0,119 \\
IMC & 18,57 & 3,53 & & 18,32 & 3,30 & 0,527 \\
\hline
\end{tabular}

DP: desvio-padrão; idade: anos; peso: quilogramas; altura: metro; S.C.: superfície corporal em m²;

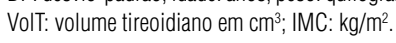

\section{Volume tireoidiano}

As medidas dos volumes tireoidianos nos sexos masculino e feminino, nas faixas etárias de 8 a 10 anos e nas três diferentes escolas (A, B e C) encontram-se na tabela 2. A análise estatística mostrou que não houve diferença considerada significante entre os sexos nessa variável. Considerando-se apenas o sexo feminino, o volume tireoidiano foi maior dos 8 para os 10 anos, quando comparadas as escolas $\mathrm{A}$ e $\mathrm{B}$ em relação à $\mathrm{C}$, e essa diferença foi estatisticamente significante $(\mathrm{p}<0,01)$. Aos 10 anos, o sexo feminino apresenta volume tireoidiano diferente nas três escolas $(\mathrm{p}<0,01)$, persistindo maior na $\mathrm{B}$ e menor na $\mathrm{C}$. Houve correlação positiva entre o volume tireoidiano e superfície corporal para todos os escolares, sendo estatisticamente significante para as meninas de 9 e 10 anos $(\mathrm{p}<0,01)$. O volume tireoidiano encontrado também se correlacionou positivamente com peso, altura e IMC. Não se observou correlação entre iodúria, ecogenicidade e volume tireoidiano. 
Tabela 2. Valores mínimo, máximo, média aritmética e respectivo desvio-padrão, do volume tireoidiano, nos meninos e meninas, nas diferentes faixas etárias

\begin{tabular}{|c|c|c|c|c|c|c|c|c|c|c|c|}
\hline \multirow{3}{*}{ Escola } & \multirow{3}{*}{ Idade } & \multicolumn{10}{|c|}{ Volume tireoidiano $\left(\mathrm{cm}^{3}\right)$} \\
\hline & & \multicolumn{5}{|c|}{ Meninos } & & \multicolumn{4}{|c|}{ Meninas } \\
\hline & & $\mathbf{n}$ & MIN & MAX & M & DP & $\mathbf{n}$ & MIN & MAX & M & DP \\
\hline \multirow[t]{3}{*}{ A } & 8 & 24 & 1,4 & 4,5 & 2,48 & 0,74 & 20 & 1,5 & 3,7 & 2,20 & 0,67 \\
\hline & 9 & 10 & 1,5 & 4,7 & 2,68 & 0,82 & 12 & 1,5 & 3,4 & 2,23 & 0,67 \\
\hline & 10 & 11 & 1,6 & 5,3 & 2,80 & 0,90 & 14 & 1,3 & 4,1 & 2,61 & 0,81 \\
\hline \multirow[t]{3}{*}{ B } & 8 & 14 & 1,6 & 4,1 & 2,83 & 0,79 & 15 & 1,5 & 4,2 & 2,38 & 0,71 \\
\hline & 9 & 12 & 2,3 & 3,7 & 3,09 & 0,67 & 20 & 1,4 & 3,8 & 2,75 & 0,90 \\
\hline & 10 & 14 & 1,9 & 4,4 & 2,97 & 0,82 & 28 & 2,3 & 5,0 & 3,71 & 0,96 \\
\hline \multirow[t]{3}{*}{ C } & 8 & 12 & 1,4 & 2,6 & 2,13 & 0,50 & 13 & 1,0 & 2,7 & 2,08 & 0,53 \\
\hline & 9 & 22 & 1,7 & 4,1 & 2,29 & 0,82 & 8 & 1,5 & 2,9 & 2,12 & 0,53 \\
\hline & 10 & 19 & 1,1 & 4,4 & 2,48 & 0,79 & 32 & 1,4 & 4,5 & 2,59 & 0,93 \\
\hline Total & 138 & & & & & & 162 & & & & \\
\hline
\end{tabular}

cm³: centímetros cúbicos; n: número de casos; MIN: valor mínimo; MAX: valor máximo; M: média; DP: desvio-padrão.

O exame ultrassonográfico da tireoide dos escolares revelou hipoecogenicidade em $11,2 \%$ das crianças da escola A, em $21,4 \%$ da B e em $8,4 \%$ da C. Foram encontrados 4 cistos ( 1 cisto em escolar da escola B e 3 cistos em escolares da escola C) e 1 nódulo sólido (hipoecoico de $7 \mathrm{~mm}$ com calcificação em lobo direito) em uma aluna da escola $\mathrm{B}$, que está em acompanhamento.

\section{lodúria}

Entre as 300 amostras urinárias analisadas, 100\% delas apresentaram valores de iodúria maiores que 100 ug/L; 24 amostras $(8,0 \%)$ com iodúria entre 100 e $200 \mathrm{ug} / \mathrm{L} ; 102$ amostras $(34 \%)$ com valores entre 200 e $300 \mathrm{ug} / \mathrm{L} ; 135$ amostras $(45,0 \%)$, entre 300 e 400 ug/L e 39 amostras (13,0\%), entre 400 e 500 ug/L. As iodúrias medianas dos escolares de Ribeirão Preto estão representadas na tabela 3 . A análise estatística mostrou diferença significante entre os meninos das escolas A, $\mathrm{B}$ e $\mathrm{C}(\mathrm{p}=0,0001$ e $\mathrm{p}=0,0106$; aos 8 e aos 10 anos, respectivamente) e entre as meninas $(\mathrm{p}=0,0024$ e $\mathrm{p}=$ 0,0154 ; aos 8 e 9 anos, respectivamente).

\section{Análise do teor de iodo em sal de uso doméstico}

Foram analisadas 205 amostras de sal de uso doméstico. A concentração mediana de iodo por quilograma de sal, os valores mínimos e máximos observados e o número de amostras abaixo de $20 \mathrm{mg} / \mathrm{kg}$ e acima de $60 \mathrm{mg} / \mathrm{kg}$ de iodo para as diferentes escolas encontram-se na tabela 4 . Vinte e sete amostras (13,2\%) apresentaram teor abaixo de $20 \mathrm{mg} / \mathrm{kg}$ e uma delas com $8,4 \mathrm{mg}$ por quilo de sal. Houve seis amostras $(2,9 \%)$ acima de $60 \mathrm{mg} /$ $\mathrm{kg}$, sendo duas com mais de $100 \mathrm{mg}$ de iodo $/ \mathrm{kg}$ de sal.
Tabela 3. Iodúria dos escolares de Ribeirão Preto

\begin{tabular}{lcccccccc}
\hline \multirow{2}{*}{ Escola } & Idade & \multicolumn{3}{c}{ Meninos } & & \multicolumn{3}{c}{ Meninas } \\
\cline { 3 - 5 } \cline { 8 - 9 } A & & $\mathbf{n}$ & MED & DP & & $\mathbf{n}$ & MED & DP \\
& 8 & 24 & 380,19 & 62,61 & & 20 & 334,84 & 81,44 \\
& 9 & 14 & 349,33 & 76,88 & & 12 & 327,26 & 81,33 \\
& 10 & 12 & 353,18 & 40,93 & & 14 & 301,56 & 88,07 \\
B & 8 & 10 & 311,78 & 93,44 & & 15 & 324,27 & 80,24 \\
& 9 & 12 & 295,68 & 82,36 & & 20 & 303,99 & 78,55 \\
& 10 & 22 & 323,83 & 62,38 & & 28 & 305,22 & 90,31 \\
C & 8 & 11 & 270,92 & 79,91 & & 13 & 252,20 & 72,93 \\
& 9 & 14 & 307,94 & 69,61 & & 8 & 267,16 & 64,50 \\
& 10 & 19 & 283,07 & 90,06 & & 32 & 279,07 & 72,06 \\
& & 138 & & & & 162 & & \\
\hline
\end{tabular}

ug/L: micrograma por litro; n: número de casos; MED: mediana; DP: desvio-padrão.

Tabela 4. Concentração (mg/kg) de iodo em 205 amostras de sal de uso doméstico entregues por crianças de três escolas de Ribeirão Preto

\begin{tabular}{lccccccc}
\hline & $\mathbf{n}$ & $\begin{array}{c}\mathbf{<} \mathbf{2 0} \\
\mathbf{m g} / \mathbf{k g}\end{array}$ & $\begin{array}{c}\mathbf{>} \mathbf{6 0} \\
\mathbf{m g} \mathbf{k g}\end{array}$ & MED & DP & Mínimo & Máximo \\
\hline Escola A & 59 & 3 & 3 & 26,69 & 15,2 & 15,04 & 125,25 \\
Escola B & 75 & 15 & 2 & 26,87 & 11,0 & 8,42 & 63,55 \\
Escola C & 71 & 9 & 1 & 27,88 & 9,40 & 12,10 & 61,03 \\
Total & 205 & 27 & 6 & & & & \\
\hline
\end{tabular}

n: número de casos; mg: miligramas; kg: quilogramas; MED: mediana, DP: desvio-padrão.

Não se observou diferença estatisticamente significante entre as concentrações medianas de iodo em amostras de sal de uso doméstico entregues pelos escolares nas três diferentes escolas.

\section{DISCUSSÃO}

No presente trabalho, realizado em $2007 / 2008$, foram observadas $59,5 \%$ das iodúrias coletadas com valores acima de $300 \mathrm{ug} / \mathrm{L}$, limite recomendado pela OMS 
$(30,31)$. Nos meninos e nas meninas, a iodúria foi mais elevada na escola A e menor na C, que está localizada em região mais periférica do perímetro urbano de Ribeirão Preto, corroborando os achados de Esteves e cols. (32) e reafirmando resultados do estudo anterior (21).

O conteúdo iódico mediano em amostras de sal para consumo trazidas pelas crianças das três escolas pesquisadas foi menor na escola B, seguida da escola C e A, embora essas diferenças não tenham sido significantes. Em torno de $13,1 \%$ das amostras de sal trazidas para análise apresentaram conteúdo iódico abaixo de 20 $\mathrm{mg} / \mathrm{kg}$ (valor recomendado) (33). Um programa de iodação do sal para uso doméstico é considerado adequado quando mais de $90 \%$ das amostras, no nível do consumidor, encontram-se com teores mínimos (34). Assim, os autores e outros trabalhos mostram que ter tornado a iodação do sal excessiva não tem garantido que o sal chegue corretamente iodado aos escolares de Ribeirão Preto.

Como a iodúria é alta em grande porcentagem da amostra e a principal fonte de ingestão de iodo é o sal iodado, muito provavelmente essa população consome grande quantidade de sal ao dia, incluindo o consumo dos alimentos industrializados e ainda, provavelmente, essas crianças têm uma ingestão de iodo diferente de acordo com o nível econômico.

$\mathrm{O}$ volume tireoidiano dos escolares avaliados foi proporcional às variáveis antropométricas, como já descrito na literatura. As crianças da escola A eram mais altas que as crianças da escola B e estas eram mais altas que as da escola $\mathrm{C}$, bem como o volume tireoidiano. No sexo feminino, essas diferenças encontradas foram mais significativas $(\mathrm{p}=0,01)$ que as encontradas no sexo masculino $(\mathrm{p}=0,04)$.

Das 300 crianças examinadas, $15,6 \%$ apresentaram hipoecogenicidade tireoidiana à ultrassonografia, sem correlação estatística com variação volumétrica da glândula tireoide ou iodúria. Dados de estudo mostram que a diminuição da ecogenicidade do parênquima glandular tireoidiano pode ser considerada sinal precoce de disfunção tireoidiana (35). Camargo e cols. observaram alterações tireoidianas na população exposta a excesso de iodo na cidade de São Paulo (36).

Os achados ultrassonográficos tireoidianos encontrados nos escolares ribeiraopretanos avaliados podem estar relacionados com exposição prévia a quantidades excessivas de iodo. Sabendo-se que o excesso de iodo nutricional é um fator ambiental importante no aumento da prevalência de tireoidite crônica, conside- rando sua intensidade e duração, sugere-se que as alterações estruturais da glândula tireoide (hipoecogenicidade) encontradas, ainda que ocorram em longo prazo quando comparadas às outras variáveis de medidas, especialmente a iodúria, possam estar relacionadas. $\mathrm{Na}$ avaliação realizada nessa cidade em 2003 , encontraram-se $20,2 \%$ de título antitireoperoxidase positivo entre as crianças examinadas. Com a oferta excessiva de iodo, a tireoglobulina passa ser mais iodada, o que a torna mais antigênica e potencialmente capaz de desencadear o processo de agressão glandular.

Mesmo que em 2003 tivesse havido orientação oficial para a mudança na concentração de iodo no sal de uso doméstico, o sal com teor de iodo mais elevado permaneceu ainda por um período sendo ofertado à população.

Neste estudo, os escolares de Ribeirão Preto encontram-se em área iodossuficiente, tendo em vista os níveis de excreção de iodo urinário encontrados. A grande maioria das amostras de sal avaliadas está adequada nos teores de iodo $/ \mathrm{kg}$ de sal. Foi observada ingestão excessiva de iodo nos escolares avaliados. Nesta amostra, a hipoecogenicidade poderia relacionar-se com alterações tireoidianas caso houvesse a avaliação da função dessa glândula e dosagem de anticorpos antitireoperoxidase, necessários para determinar melhor o grau de comprometimento tireoidiano nesse grupo.

Agradecimentos: Este trabalho não teria sido realizado sem o precioso auxílio de Daiane Roza e do professor Dr. Edson Z. Martinez do CEMEQ - FMRP-USP, do professor Dr. Manoel Henrique Gabarra da Estatística da UNAERP, dos alunos da Liga de Endocrinologia da Universidade de Ribeirão Preto, na ajuda da coleta do material, dos alunos Ramon Roncaratti Nicotari, Stéphane de Bonifácio, Carla Fernanda Trevisan, Mariana de Souza Morando e Mariana Melegari Arnarante do curso de Nutrição da Universidade de Ribeirão Preto, nas coletas e dosagens das iodúrias, da Sra. Wilma Tomimori e de Antonio Felipe Alves Cortes, no auxílio em várias etapas do trabalho.

Declaração: os autores declaram não haver conflitos de interesse científico neste estudo.

\section{REFERÊNCIAS}

1. Blum M, Goldman AB, Herskovic A, Hemberg J. Clinical applications of thyroid echography. N Engl J Med. 1972;287:1164-9.

2. Blum M, Yee J. Advances in thyroid imaging: thyroid sonography-when how should it be used? Thyroid Today. 1997;29:1-13.

3. Berghout A, Wiersinga WM, Smits NJ, Touber JL. The value of thyroid volume measured by ultrasonography in the diagnosis of goiter. Clin Endocrinol (Oxf). 1988;28(4):409-14.

4. Vitti P, Martino E, Anghini-Lombardi F, RagoT, Antonangeli L, Maccherini $D$, et al. Thyroid volume measurement by ultrasound in 
children as a tool for the assessment of mild iodine deficiency. J Clin Endocrinol Metab. 1994;79:600-3.

5. Ueda D. Normal volume of the thyroid gland in children. J Clin Ultrasound. 1990;18:455-62.

6. Elementos traço na nutrição e saúde. Organização Mundial da Saúde (OMS); 1998.

7. Lamberger BA. lodine deficiency disorders and endemic goiter. Europ Clin Nutr. 1993;47:1-8.

8. Delange $F$. The disorders induced by iodine deficiency. Thyroid. 1994;4(1):107-28.

9. Fisher DA. Efectiveness of newborn screening programs for congenital hypothyroidism: prevalence of missed cases. Pediatr Clin North Am. 1987;34:881-90.

10. Delange F, Bourdoux P, Ermans AM. Transient disorders of thyroid function and regulation in preterm infants. In: Delange $F$, Fisher DA, Malvaux $\mathrm{P}$, editors. Pediatric thyroidology. Basel: Karger. 1985, p. 369-93.

11. Delange $F$, Heidemann $P$, Bourdoux $P$, Larsson A, Vigneri $R$, Klett $M$, et al. Regional variations of iodine nutrition and thyroid function during the neonatal period in Europe. Biol Neonate. 1986;49:322-30.

12. Hetzel BS. lodine deficiency disorders (IDD) and their eradication. Lancet. 1983;2(8359):1126-9.

13. Boyages $\mathrm{S}$. Thyroid autoimmunity in edemic goiter caused by excessive iodine intake. Clin Endocrinol. 1998;31:453-65.

14. Furnee CA, West CE, Van der Haar F, Hautvast JV. Efficacy of oral iodised oil is associated with anthropometric status in severely iodine-deficient schoolchildren in rural Malawi. $\mathrm{Br} \mathrm{J}$ Nutr. 2000;84(3):345-52.

15. Zhao J, Wang P, Shang L, Sullivan KM, Van der Haar F, Malverly G. Endemic goiter associated with high iodine intake. Am J Publ Health. 2000;90(10):1633-5.

16. Agência Nacional de Vigilância Sanitária. Internet: Disponível em: <http: elegis.anvisa.gov.br// leis ref/ public showAct.php>. Acessado em: 17 Maio 2010.

17. Lei Federal 6.150, de 03/12/1074: Dispõe sobre a obrigatoriedade da iodação do sal destinado ao consumo humano, seu controle pelos órgãos sanitários e outras providências.

18. Duarte GC, Tomimori EK, Boriolli RA, Ferreira JE, Catarino RM, Camargo YA, et al. Avaliação ultrassonográfica da tireoide e determinação da iodúria em escolares de diferentes regiões do estado de São Paulo. Arq Bras Endocrinol Metab. 2004;48(6):842-8.

19. Duarte GC, Tomimori EK, Camargo RYA, Rubio IGS, Wajngarten $M$, Rodrigues AG, et al. The prevalence of thyroid dynfunction in elderly cardiology patients with mild excessive iodine intake in the urban área of São Paulo. Clinics. 2009;64(2):135-42.
20. Alves MLD, Maciel RMB, Kunii I, lazigi N. Correlação entre níveis de iodúria eTSH colhido em cordão umbilical de recém-nascidos do Hospital das Clínicas da Faculdade de Medicina de Ribeirão Preto, São Paulo. Arq Bras Endocrinol Metab. 2005;49(4):516-20.

21. Alves MLD, Scandiuzzi SAM, Pelizzari LA, Jordão AM, Ramos APP, Navarro AM. Correlação entre níveis de iodúria, presença de bócio e título de anticorpos antitireoidianos entre escolares de Ribeirão Preto. Arq Bras Endocrinol Metab. 2006;50(3) S1, PO 154: 357.

22. Brunn J, Block U, Ruf G, Bos I, Kunze WP, Scriba PC. Volumetric analisys of thoroid lobes by real-time ultrasound. Dtsch Med Wochenschr. 1981;106(41):1338-40.

23. Sandell EB, Kolthoff IM. Microdetermination of iodine by a catalytic method. Mikrovekemica Acta. 1937;1:9-25.

24. Pino S, Fang SL, Barverman LE. Ammonium persulfate: a safe alternative oxidizing reagent for measuring urinary iodine. Clin Chem. 1996;42(2):239-43.

25. Dunn JT, Crutchfield He, Gutekunst R, Dunn AD. Methods for measuring iodine in urine. International Council for Control of lodine Deficiency disorders. Wageningen: ICCIDD; 1993.

26. Montgomery DC. Design and analysis of experiments. 5. ed. New York: John Wiley \& Sons, Inc.; 2000.

27. SAS/STAT ${ }^{\oplus}$ User's guide, Version 9.1, Cary, NC: SAS Institute Inc.; 2002.

28. Snedecor GN, CoChran WG. Statical methods. 6. ed. lowa: The lowa State University Press; 1972.

29. Pagano M, Gawreau K. Correlação. In: Princípios de bioestatística. São Paulo: Pioneira Thomson Learning. 2. ed. traduzida; 2004, p. 352-66.

30. Dunn JT. Guarding our nation's thyroid health. J Clin Endocrinol Metab. 2002;87:486-8.

31. Knobel $M$, Medeiros-Neto $G$. Moléstias associadas à carência crônica de iodo. Arq Bras Endocrinol Metab. 2004;48(1):53-61.

32. Esteves RZ, KasamatsuTS, Kunii IS, Furuzawa GK, Vieira JGH, Maciel RMB. Desenvolvimento de um método para a determinação da iodúria e sua aplicação na excreção urinária de iodo em escolares brasileiros. Arq Bras Endocrinol Metab. 2007;51(9):1477-84.

33. Medeiros-Neto G. lodine nutrition in Brazil: where do we stand? Arq Bras Endocrinol Metab. 2009;53(4):470-4.

34. Indicators for assessing iodine deficiency disorders and their control program. Review version, WHO/NUT/93.1. WHO, Geneva; 1994.

35. Camargo RYA, Tomimori EK. Programa de atualização em doenças da tireoide: ultrassonografia da tireoide, fascículo 4, SBEM.

36. Camargo RY, Tomimori EK, Neves SC, Rubio GS, Galrão Al, Knobel $M$, et al. Thyroid and the environment exposure to excessive nutricional iodine increases the prevalence of thyroid disorders in São Paulo, Brazil. Eur J Endocrinol. 2008;159(3);293-9. 